Pacific Journal of Mathematics

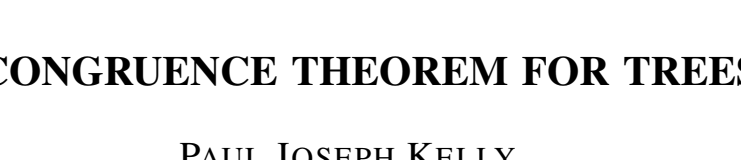




\title{
A CONGRUENCE THEOREM FOR TREES
}

\author{
PAUL J. KeLLY
}

Let $A$ and $B$ be two trees with vertex sets $a_{1}, a_{2}, \cdots, a_{n}$ and $b_{1}, b_{2}, \cdots, b_{n}$ respectively. The trees are congurent, are isomorphic, or "are the same type", $(A \cong B)$, if there exists a one-to-one correspondence between their vertices which preserves the join-relationship between pairs of vertices. Let $c\left(a_{i}\right)$ denote the $(n-1)$-point subgraph of

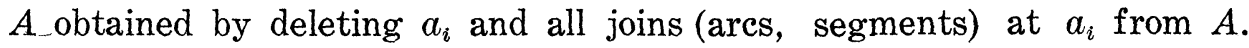
It is the purpose here to show that if there is a one-to-one correspondence in type, and frequency of type, between the sub-graphs of order $n-1$ in $A$ and $B$, that is, if there exists a labeling such that $c\left(a_{i}\right) \cong$ $c\left(b_{i}\right), i=1,2, \cdots, n$, then $A \cong B$. It is assumed throughout, therefore, that there is a labeling of the two trees $A$ and $B$ such that $c\left(a_{i}\right) \cong c\left(b_{i}\right)$, $i=1,2, \cdots, n$, where $n \geqq 3$.

Some lemmas to the main theorem are established first. Let $T$ denote a certain type of graph of order $j$, where $2 \leqq j<n$, which occurs as a subgraph $\alpha$ times in $A$ and $\beta$ times in $B$. If $\alpha_{i}$ is the number of $T$-type subgraphs which have $a_{i}$ as a vertex, then,

$$
\alpha=\left(\sum_{1}^{n} \alpha_{i}\right) / j
$$

Similarly,

$$
\beta=\left(\sum_{1}^{n} \beta_{i}\right) / j,
$$

where $b_{i}$ is the number of $T$-type subgraphs having $b_{i}$ as a vertex. Because $c\left(a_{i}\right) \cong c\left(b_{i}\right)$, the number of $T$-type subgraphs which do not have $a_{i}$ as a vertex is the same as the number which do not have $b_{i}$ as a vertex. Thus

$$
\alpha-\alpha_{i}=\beta-\beta_{i}, \quad i=1,2, \cdots, n .
$$

Therefore

$$
\sum_{1}^{n}(\alpha-\beta)=\sum_{1}^{n}\left(\alpha_{i}-\beta_{i}\right),
$$

so $n(\alpha-\beta)=j(\alpha-\beta)$, which implies $\alpha=\beta$. This, in turn, implies $\alpha_{i}=\beta_{i}$, $i=1,2, \cdots, n$, and the lemma is established.

Lemma 1. Every type of proper subgraph which occurs in $A$ or $B$ Received December 16, 1955. 
occurs the same number of times in both, and $a_{i}$ and $b_{i}$ are vertices in the same number of these subgraphs, $i=1,2, \cdots, n$.

The case $j=2$ gives a special result.

Lemma 2. The vertices $a_{i}$ and $b_{i}$ have the same degree, $i=1,2, \cdots, n$.

Next it is clear that if either $A$ or $B$ consists of just a path between two end points then the other is also a path of the same length. If neither is just a path, then their maximal-length paths are proper subgraphs and have the same length because of Lemma 1.

This proves the third lemma.

Lemma 3. The trees $A$ and $B$ have the same radius $r$ and both trees are central or both are bicentral.

A correspondence between $c\left(a_{i}\right)$ and $c\left(b_{i}\right)$, under which $c\left(a_{i}\right) \cong c\left(b_{i}\right)$, will be called an $a_{i}$-mapping (or $b_{i}$-mapping), and the main theorem is obtained by using these submappings to define a congruence of $A$ and $B$. Because such a congruence is more easily obtained when the trees are central, the proof will be carried through for bicentral trees only, with the simpler proof implied by analogy. It is supposed therefore that $A$ has bicenters $\bar{a}_{1}$ and $\bar{a}_{2}$ and that $B$ has bicenters $\overline{b_{1}}$ and $\overline{b_{2}}$ (where $\bar{a}_{1}$ is not necessarily $a_{1}$ ).

Let $F$ be a component in the graph obtained by deleting from $A$ the bicenters and all joins to them. There is a point of $F$ joined in $A$ to one bicenter, say $\bar{a}_{1}$, and no point of $F$ is joined in $A$ to $\bar{a}_{2}$. By $(\bar{a} \cup F)$ is meant the graph, which has for its vertices $\bar{a}$, and the vertices of $F$, and whose joins are the same as they are in $A$. The graph $\left(\bar{a}_{1} \cup F\right)$ is a limb at $\bar{a}_{1}$. It is a radial or nonradial limb according as it does not possess an $r$-point, that is, a point whose distance in $A$ from the nearest bicenter is $r$. An easy consequence of Lemma 1 is that $a_{i}$ is an $r$-point if and only if $b_{i}$ is an $r$-point.

Some special subgraphs of $A$ and $B$ are now defined. At $\bar{a}_{i}$ the radial limbs are

$$
A_{i 1}, A_{i 2}, \cdots, A_{i m_{i}},
$$

and the non-radial limbs are

$$
C_{i 1}, C_{i 2}, \cdots, C_{i s_{i}},
$$

while at $\overline{b_{i}}$ the radial limbs are

$$
B_{i 1}, B_{i 2}, \cdots, B_{i n_{i}}
$$


and the non-radial limbs are

$$
D_{i 1}, D_{i 2}, \cdots, D_{i t_{\imath}}, \quad i=1,2 .
$$

Next,

$$
\begin{gathered}
A_{i}=\left(A_{i 1} \cup A_{i 2} \cup \cdots \cup A_{i m_{i}}\right), \quad B_{i}=\left(B_{i 1} \cup B_{i 2} \cup \cdots \cup B_{i m_{i}}\right), \\
C_{i}=\left(C_{i 1} \cup C_{i 2} \cup \cdots \cup C_{i s_{i}}\right),
\end{gathered}
$$

and

$$
D_{i}=\left(D_{i 1} \cup D_{i 2} \cup \cdots \cup D_{i t_{i}}\right), \quad i=1,2
$$

Finally,

$$
A_{r}=\left(A_{1} \cup A_{2}\right), \quad B_{r}=\left(B_{1} \cup B_{2}\right), C=\left(C_{1} \cup C_{2}\right),
$$

and

$$
D=\left(D_{1} \cup D_{2}\right)
$$

In obtaining congruences for these special subgraphs, an important role is played by center preserving mappings, that is, those which pair $\bar{a}_{1}$ and $\bar{a}_{2}$ in some order with $\bar{b}_{1}$ and $\bar{b}_{2}$. It is useful, therefore, to define a vertex $a_{i}$ to be a nonessential point, (n. e. point), if it is of degree one (is an end point) such that $c\left(a_{i}\right)$ is a bicentral tree of radius $r$. Every end point, which is not an $r$-point, is an n.e. point. An $r$-point is nonessential if it belongs to a limb with more than one $r$ point, or if the bicenter to which its limb belongs has more than one radial limb. If $a_{i}$ is an n.e. point then $b_{i}$ is an n. e. point and every $a_{i}$-mapping is center preserving. The following fact is also useful.

Lemma 4. If $a_{i}$ is an n. e. point of $A$ in $A_{r}$ then $b_{i}$ is an n. e. point of $B$ in $B_{r}$.

Proof. Assume $b_{i} \notin B_{r}$, that is, $b_{i} \in D$. Any $a_{i}$-mapping must pair the remainder of $A_{r}$ (without $a_{i}$ ) with all of $B_{r}$, so the order of $A_{r}$ is one greater than that of $B_{r}$. If $A$ had a nonradial limb it would have an n. e. point in $C$, say $a_{j}$, and an $a_{j}$-mapping would have to pair $A_{r}$ with all or part of $B_{r}$, which is impossible. Therefore $A$ has no nonradial limb and $b_{i}$ is the only point of $B$ not in $B_{r}$. The sum of the degrees of $\bar{a}_{1}$ and $\bar{a}_{2}$ is therefore smaller than the sum of the degrees of $\bar{b}_{1}$ and $\bar{b}_{2}$. If $B$ had an n. e. point $b_{l}$, distinct from $b_{i}$, the sum of the degrees of $\overline{b_{1}}$ and $\overline{b_{2}}$ would be the same in $c\left(b_{l}\right)$ as in $B$, and therefore a $b_{l}$-mapping could not be center preserving. From this it follows that $a_{i}$ and $b_{i}$ are the only n. e. points in $A$ and $B$ respectively. Thus 
$A$ consists of a $(2 r+1)$-path and one extra point $a_{i}$ joined to a point $a_{k}$, which is not a center, while $B$ consists of a $(2 r+1)$-path and one extra point $b_{i}$ joined to a center. This center is $b_{k}$ since $a_{k}$ is the only point in $A$ of degree three. But now it is clear that $c\left(a_{k}\right)$ has a component which is a path of greater length than any in $c\left(b_{k}\right)$, which contradicts $c\left(a_{k}\right) \cong c\left(b_{k}\right)$. The assumption that $b_{i}$ is in $D$ is therefore false and Lemma 4 is established.

Theorem. If $A$ and $B$ are trees with vertices $a_{1}, a_{2}, \cdots, a_{n}$ and $b_{1}, b_{2}, \cdots, b_{n}, n \geq 3$, respectively. and $c\left(a_{i}\right) \cong c\left(b_{i}\right), i=1,2, \cdots, n$ then $A \cong B$.

Proof. As previously indicated, the details will be given only for the case where $A$ and $B$ are bicentral.

Case 1. One of the trees, say $A$, has a nonradial limb. Then $A$ has an n.e. point $a_{k}$ in $C$ and, from Lemma $4, b_{k}$ is in $D$. An $a_{k}$ mapping, therefore, pairs $A_{r}$ with $B_{r}$, so

$$
A_{r} \cong B_{r} \text {. }
$$

Next,

(2) There is a congruence of $C$ and $D$ which pairs $\bar{a}_{1}, \bar{a}_{2}$ in some order with $\overline{b_{1}}$ and $\overline{b_{2}}$.

Consider the n. e. points of $A$ in $A_{r}$. First, suppose the limb to which one of these points $a_{i}$ belongs is still of length $r$ after $a_{i}$ is deleted from it. Then an $a_{i}$ mapping cannot take this sub-limb into $D$, and so must pair the remainder of $A_{r}$ with the remainder of $B_{r}$. It therefore pairs $C$ with $D$ as stated in (2). Next, suppose every n.e. point which belongs to $A_{r}$ or $B_{r}$ is the end of an $r$-path limb. If $a_{i}$ and $b_{i}$ are such points, then deleting them from their limbs produces two $(r-1)$ path limbs. Since these sub-limbs are congruent, an $a_{i}$-mapping either pairs $C$ and $D$ as stated in (2) or else can be redefined to do so. The only remaining possibility is that no n.e. point occurs in either $A_{r}$ or $B_{r}$, so each is a $(2 r+1)$-path. Let $a_{i}$ be the $r$-point in $A_{i}$ and $b_{i}$ be the $r$-point in $B_{i}, i=1,2$. Since $C\left(a_{1}\right)$ is a tree of radius $r$ and center $\bar{a}_{2}$ and $c\left(b_{1}\right)$ is a congruent tree with center $\bar{b}_{2}$, an $a_{1}$-mapping pairs $\bar{a}_{2}$ and $\overline{b_{2}}$. It must also pair the nonradial limbs of $A$ at $\bar{a}_{2}$ with the nonradial limbs of $B$ at $\overline{b_{2}}$, and hence $C_{2} \cong D_{2}$. By the same reasoning, an $a_{2^{-}}$ mapping establishes a congruence of $C_{1}$ and $D_{1}$ which pairs $\bar{a}_{1}$ with $\bar{b}_{1}$, so there is clearly a congruence of $C$ and $D$ satisfying (2).

If a congruence of $C$ and $D$, satisfying (2), and a congruence of 
$A_{r}$ and $B_{r}$ both pair the bicenters in the same order, then clearly $A \cong$ $B$. Assume, on the contrary, that every congruence of $A_{r}$ with $B_{r}$ pairs the bicenters in one order, say $\bar{a}_{1}$ with $\bar{b}_{1}$ and $\bar{a}_{2}$ with $\bar{b}_{2}$, while every congruence of $C$ and $D$, satisfying (2), pairs the bicenters in the opposite order, namely $\bar{a}_{1}$ with $\bar{b}_{2}$ and $\bar{a}_{2}$ with $\bar{b}_{1}$. It will be shown that this leads to a contradiction and hence that $A \simeq B$ under Case 1. First, from the assumption about the congruence of $A_{r}$ and $B_{r}$, it follows that each is not just a $(2 r+1)$-path. Therefore $A$ has an n. e. point $a_{i}$ in $A_{r}$, and it may be supposed that $a_{i} \in A_{1}$. Since an $a_{i}$-mapping implies a congruence of $C$ and $D$ satisfying (2) it must pair $\bar{a}_{1}$ and $\bar{a}_{2}$ with $\bar{b}_{2}$ and $\overline{b_{1}}$ in that order. By assumption, $A_{1} \cong B_{1}$ and $A_{2} \cong B_{2}$, so $b_{i} \in B_{2}$ would imply that an $a_{i}$-mapping pairs $A_{2}$ with $B_{1}$. But then $A_{1} \cong A_{2} \cong B_{1} \cong B_{2}$ would contradict the unique mapping of bicenters in any congruence of $A_{r}$ with $B_{r}$. Therefore $b_{i} \in B_{1}$. Let $f_{1}$ be the order of $A_{1}$ and $B_{1}$ and $f_{2}$ be that of $A_{2}$ and $B_{2}$. An $a_{i}$-mapping shows $f_{2}=f_{1}-1$. Suppose $A$

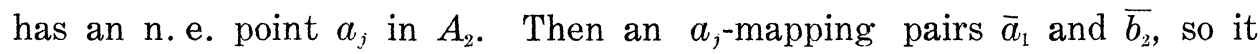
pairs $A_{1}$ with all or part of $B_{2}$. But this is impossible because $f_{1}>f_{2}$. Therefore there is no n. e. point of $A$ in $A_{2}$, and, by the same reasoning, there is no n.e. point of $B$ in $B_{2}$. Thus $A_{2}$ and $B_{2}$ are paths of length $r$, and $A$ and $B$ each have just two end points in $A_{1}$ and $B_{1}$ respectively.

Now consider nonradial limbs. At least one exists so, from Lemma 4 , at least one each exists in each tree. Suppose there is a nonradial jimb at $a_{1}$, and let $a_{j}$ be an end point of $A$ in this limb. Then $b_{j} \in D$. Because an $a_{j}$-mapping includes a congruence of $A_{r}$ and $B_{r}$, it pairs $a_{1}$ with $\bar{b}_{1}$ and $\bar{a}_{2}$ with $\bar{b}_{2}$. If $b_{j}$ were in $D_{1}$ such a mapping would imply $C_{2} \cong D_{2}$, and this, with $C_{1} \cong D_{2}$ and $C_{2} \cong D_{1}$, would yield $C_{1} \cong C_{2} \cong D_{1}$ $\cong D_{2}$, contradicting the unique center pairings in a congruence of $C$ and $D$. Therefore $b_{j} \in D_{2}$. Let $f_{3}$ be the order of $C_{1}$ and $D_{2}$ and $f_{4}$ by the order of $C_{2}$ and $D_{1}$. An $a_{j}$-mapping shows that $f_{3}-1=f_{4}$. Therefore there is no n.e. point in $C_{2}$ and none in $D_{1}$. For if $a_{k}$ in $C_{2}$ were an n. e. point, an $a_{k}$-mapping would pair $\bar{a}_{1}$ with $\bar{b}_{1}$ and therefore would pair $C_{1}$ with all or part of $D_{1}$. This is impossible because $f_{3}>f_{4}$. There are, therefore, no nonradial limbs at $\bar{a}_{2}$ or $\bar{b}_{1}$, and there is just one nonradial limb at $\bar{a}_{1}$ and at $b_{2}$, each of length one. The center $\bar{a}_{1}$ and $\bar{b}_{2}$ are of degree three and $\bar{a}_{2}$ and $\bar{b}_{1}$ have degree two. Let $a_{1}$ be the end point of $A$ in $A_{2}$. The tree $c\left(a_{1}\right)$ has only one center, namely $\bar{a}_{1}$ of degree three. If the $r$-point $b_{1}$ were in $B_{2}$, the center of $c\left(b_{1}\right)$ would have degree two, contradicting $c\left(a_{1}\right) \simeq c\left(b_{1}\right)$. So $b_{1} \in B_{1}$. Also $b_{1}$ is the only $r$-point in $B_{1}$, for otherwise $c\left(b_{1}\right)$ would be a bicentral tree. If $a_{22}$ and $b_{2}$ denote the other $r$-points of $A$ and $B$ respectively, it fol- 
lows that $c\left(a_{2}\right)$ and $c\left(b_{2}\right)$ are central trees and that $\bar{a}_{2}$ and $\bar{b}_{1}$ are their respective centers. But in $c\left(a_{2}\right)$ the radial arm to $a_{1}$ is a path in $c\left(b_{2}\right)$ both radial arms branch, and this contradicts $c\left(a_{2}\right) \cong c\left(b_{2}\right)$. The supposition that a nonradial limb exists at $\bar{a}_{2}$ rather than $\bar{a}_{1}$ leads to the same kind of contradiction, hence $A \cong B$ under all the possibilities of Case 1 .

Case 2. There are no nonradial limbs but one tree has at least three radial limbs. Suppose there are at least two radial limbs at $\bar{a}_{2}$, and now let $\bar{a}_{1}=a_{1}$ and $\bar{b}_{2}=b_{2}$. One and only one component of $c\left(a_{1}\right)$ is a central tree of radius $r$. Its center is $\bar{a}_{2}$ and all of its limbs are radial. Let $b_{1}^{\prime}$ be the center of the corresponding, congruent tree in $c\left(b_{1}\right)$. If $b_{1}$ is neither $\bar{b}_{1}$ or $\bar{b}_{2}$, then $b_{1}^{\prime}$ is a non-end point of $B$ in some limb of $B$, say a limb at $\overline{b_{1}}$. There is a path $P$ from $\bar{b}_{1}$, to $b_{1}^{\prime}$ and there also exists a path $P^{\prime}$ starting at $b_{1}^{\prime}$ and having no join in common with $P$. Since the length of $P^{\prime}$ must be less than $r$, all the limbs of the tree centered at $b_{1}^{\prime}$ cannot be radial. The supposition that $b_{1}$ is neither $\overline{b_{1}}$ or $\overline{b_{2}}$ is therefore false, and $b_{1}$ may be taken to be $\bar{b}_{1}$. Then $A_{2} \cong B_{2}$ is implied by an $a_{1}$-mapping.

If there are at least two radial arms at either $a_{1}$ or $b_{1}$, the same reasoning shows that $A_{1} \cong B_{1}$ and this, with $A_{2} \cong B_{2}$, implies $A \cong B$. Suppose, then, that $A_{11}$ and $B_{11}$ are the only limbs at $a_{1}$ and $b_{1}$ respectively, and let the order of $A_{11}$ be at least as great as that of $B_{11}$. There is an $r$-point $a_{j}$ in $A_{21}$ and it is an n.e. point. An $a_{j}$-mapping must pair $a_{1}$ with $b_{1}$ because these are of degree two while $\bar{a}_{22}$ and $\bar{b}_{2}$ are of degree at least three. The mapping therefore pairs $A_{11}$ with all or part of $B_{11}$, and since the latter case is excluded by the orders of $A_{11}$ and $B_{11}$, it follows that $A_{11} \cong B_{11}$. This, with $A_{2} \cong B_{2}$, implies $A \cong B$ and completes Case 2.

Case 3. Each tree has exactly two limbs. Let $n_{i}$ be the order of $A_{i}$ and $n_{i}^{\prime}$ be the order of $B_{i}, i=1,2$. Assume that the pair $n_{1}, n_{2}$ is not the pair $n_{1}^{\prime}, n_{2}^{\prime}$ in either order. Then, because $n_{1}+n_{2}=n_{1}^{\prime}+n_{2}^{\prime}$, one of the four numbers is a strict maximum. Suppose $n_{2}>\max \left(n_{1}, n_{1}^{\prime}, n_{2}^{\prime}\right)$. Then $A_{2}$ is not congruent to $B_{1}$ or $B_{2}$ or any of their subgraphs, and therefore $A_{1}$ has no n. e. points. It is therefore a path with one $r$ point of $A$, say $a_{3}$. Then vertex $b_{3}$ is an $r$-point and is the only $r$-point of its limb because $a_{3}$ is not an n. e. point. The tree $c\left(a_{3}\right)$ is central, has radius $r$, and $\bar{a}_{2}$ is its center, so its two radial limbs have orders $n_{1}$ and $n_{2}$. The center of $c\left(b_{3}\right)$ is either $\bar{b}_{1}$ or $\bar{b}_{2}$, but in either case the two limbs have orders $n_{1}^{\prime}$ and $n_{2}^{\prime}$, so a congruence of $c\left(a_{3}\right)$ and $c\left(b_{3}\right)$ is impossible. From this contradiction it follows that $n_{1}$ and $n_{2}$ are in some order the numbers $n_{1}^{\prime}$ and $n_{2}^{\prime}$ and it may be supposed that $n_{1}=n_{1}^{\prime}$ 
and $n_{2}=n_{2}^{\prime}$.

Now consider the n. e. points. If none exist, then both trees are $(2 r+1)$-paths and hence are congruent. If, on the other hand, $a_{i}$ is an n. e. point of $A$, then $b_{i}$ is an n. e. point of $B$ and the following applies :

(3) If $a_{i}$ and $b_{i}$ are n. e. points, with $a_{i}$ in $A_{1}$ and $b_{i}$ in $B_{2}$ (or $a_{i}$ in $A_{2}$ and $b_{i}$ in $B_{1}$ ), then $A \cong B$.

For, suppose $a_{i} \in A_{1}$ and $b_{i} \in B_{2}$. Then because of the orders of the limbs, an $a_{i}$-mapping pairs $A_{2}$ with $B_{1}$, so $A_{2} \cong B_{1}$ and $n_{1}=n_{2}$. If there is no n. e. point of $A$ in $A_{2}$ then $A_{2}$ is an $r$-path and so is $B_{1}$ because it is congruent to $A_{2}$. But then, because $n_{1}=n_{2}$, both $A_{1}$ and $B_{2}$ are also $r$ paths, which is contradictory. Therefore, there exists an n.e. point $a_{j}$ in $A_{2}$. Because $n_{1}=n_{2}$, an $a_{j}$-mapping pairs $A_{1}$ either with $B_{2}$ or with $B_{1}$. The first case, together with $A_{2} \simeq B_{1}$, implies $A \cong B$ directly. The second case implies $A_{1} \cong B_{1} \cong A_{2}$, and from this it follows that there is an n. e. point, say $b_{k}$, in $B_{1}$. Then a $b_{k}$-mapping pairs $B_{2}$ with either $A_{1}$ or $A_{2}$. Therefore all the limbs are the limbs are the same type and $A \cong B$.

Because of (3), it is now only necessary to consider the case $a_{i} \in A_{1}$ and $b_{i} \in B_{1}$. There are two sub-cases.

Case 3.1. There is no n. e. point in either $A_{2}$ or $B_{2}$. Then $A_{2} \simeq B_{2}$ since they are both $r$-paths. Let the end point of $A_{2}$ be $a_{3}$. Then $c\left(a_{3}\right)$ is a central tree, of radius $r$, whose center is $\bar{a}_{1}$. From $c\left(a_{3}\right) \cong c\left(b_{3}\right)$, it follows that $b_{3}$ is the only $r$-point of some limb in $B$. Assume $b_{3} \in B_{1}$. Let $b_{4}$ be the $r$-point of $B$ in $B_{2}$. Then $a_{4}$ is the only $r$-point of $A$ in $A_{1}$. An $a_{4}$-mapping pairs $\bar{a}_{2}$ with $\bar{b}_{1}$ and also pairs the limb of $c\left(a_{1}\right)$ which is not a path with the limit of $c\left(b_{4}\right)$ which is not a path. It therefore pairs $\bar{a}_{1}$ with $b_{11}$, the first point in the limb $B_{1}$. Because $\bar{a}_{1}$ is of degree two, the point $b_{11}$ is of degree two and so is joined to a well defined second point in $B_{1}$, say $b_{12}$. An $a_{3}$-mapping pairs $\bar{b}_{2}$ and $\bar{a}_{1}$, and, by the same reasoning as before, pairs $\bar{b}_{1}$ with the first point, say $a_{11}$, in $A_{1}$. Then $a_{11}$ is of degree two and so is joined to a well defined second point $a_{12}$ in $A_{1}$. An $a_{4}$-mapping must, then, pairs $a_{11}$ with $b_{12}$, so $b_{21}$ is of degree two and joins the third point in $B_{1}$. Alternating this way between the $a_{3}$ and $a_{4}$ mappings, it follows that all points of $A_{1}$ and $B_{1}$ are of degree two, which is absurd. The assumption that $b_{3}$ is in $B_{1}$ is therefore false, so $b_{3} \in B_{2}$. Now an $a_{3}$ mapping must pair $\bar{a}_{1}$ with $\bar{b}_{1}$ and must also pair the branching and non-branching limbs at $\bar{\alpha}_{1}$ and $\bar{b}_{1}$. Therefore $A_{1} \cong B_{1}$, and this, with $A_{2} \cong B_{2}$, implies $A \cong B$. 
Case 3.2. There is an n. e. point in $A_{2}$ or else there is one in $B_{2}$. Suppose $a_{j} \in A_{2}$ is nonessential. If $b_{j}$ is in $B_{1}$, then, from (3), $A \cong B$, so suppose $b_{j} \in B_{2}$. If an $a_{i}$-mapping pairs $A_{2}$ with $B_{22}$ and an $a_{j}$-mapping pairs $A_{1}$ with $B_{1}$ then clearly $A \cong B$. So suppose an $a_{i}$-mapping pairs $A_{2}$ with the remainder of $B_{1}$ (without $b_{i}$ ). Then $n_{1}=n_{2}+1$ and because of this an $a_{j}$-mapping pairs $A_{1}$ with $B_{1}$, hence $A_{1} \cong B_{1}$. Let $a_{k}$ be the point of $A_{1}$ paired with $b_{i}$ in an $a_{5}$-mapping. Then $A_{1}$ minus $a_{k}$, that is the graph obtained from $A_{1}$ by deleting $a_{k}$ and all joins to $a_{k}$, is congruent to $B_{1}$ minus $b_{i}$. But an $a_{i}$-mapping pairs $A_{2}$ with $B_{1}$ minus $b_{i}$. Therefore $c\left(a_{k}\right)$ is a bicentral tree both of whose limbs are congruent to $A_{2}$. From Lemma 1 there is a subgraph of the same type in $B$ and hence $B_{2} \cong A_{2}$. This, with $A_{1} \cong B_{1}$, implies $A \cong B$ and completes the proof.

It is natural to wonder if any two graphs must be isomorphic when they have the same composition in terms of $(n-1)$-point subgraphs. The author has considered the question for graphs having at most one join for any pair of points, with no point joined to itself. Actual inspection shows that the theorem is valid for all such graphs up to order seven. It also holds for any two such graphs of general, finite order if either is disconnected or its transpose is disconnected. (The transpose is obtained by reversing the join relationship between every pair of vertices.) However, the author was unable to prove or disprove the general case. As a final comment, it is not true that the same composition in terms of $(n-2)$-point subgraphs implies isomorphism. 


\section{PACIFIC JOURNAL OF MATHEMATICS}

EDITORS

H. L. ROYDEN

Stanford University

Stanford, California

R. A. Beaumont

University of Washington

Seattle 5 , Washington
A. R. Whiteman

University of Southern California

Los Angeles 7, California

E. G. Straus

University of California

Los Angeles 24, California

\section{ASSOCIATE EDITORS}
E. F. BECKENBACH
C. E. BURGESS
M. HALL
E. HEWITT
A. HORN
V. GANAPATHY IYER
R. D. JAMES
M. S. KNEBELMAN

L. NACHBIN

I. NIVEN

G. SZEKERES

T. G. OSTROM

M. M. SCHIFFER
F. WOLF

K. YOSIDA

\section{SUPPORTING INSTITUTIONS}

UNIVERSITY OF BRITISH COLUMBIA

CALIFORNIA INSTITUTE OF TECHNOLOGY

UNIVERSITY OF CALIFORNIA

MONTANA STATE UNIVERSITY

UNIVERSITY OF NEVADA

OREGON STATE COLLEGE

UNIVERSITY OF OREGON

UNIVERSITY OF SOUTHERN CALIFORNIA

\author{
STANFORD UNIVERSITY \\ UNIVERSITY OF UTAH \\ WASHINGTON STATE COLLEGE \\ UNIVERSITY OF WASHINGTON \\ AMERICAN MATHEMATICAL SOCIETY \\ CALIFORNIA RESEARCH CORPORATION \\ HUGHES AIRCRAFT COMPANY \\ THE RAMO-WOOLDRIDGE CORPORATION
}




\section{Pacific Journal of Mathematics}

\section{Vol. 7, No. $1 \quad$ January, 1957}

Richard Davis Anderson, Zero-dimensional compact groups of

homeomorphisms ................................... 797

Hans-Joachim Bremermann, Holomorphic functionals and complex

convexity in Banach spaces........................... 811

Hugh D. Brunk, G. M. Ewing and W. R. Utz, Minimizing integrals in

certain classes of monotone functions ................. 833

Philip David, Uniqueness theory for asymptotic expansions in general

regions ...................................... 849

Paul Erdős and Harold Nathaniel Shapiro, On the least primitive root of a

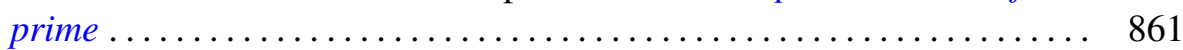

Watson Bryan Fulks, Regular regions for the heat equation ........... 867

William Robert Gaffey, A real inversion formula for a class of bilateral

Laplace transforms ................................ 879

Ronald Kay Getoor, On characteristic functions of Banach space valued random variables ................................. 885

Louis Guttman, Some inequalities between latent roots and minimax (maximin) elements of real matrices ...................... 897

Frank Harary, The number of dissimilar supergraphs of a linear graph .... 903

Edwin Hewitt and Herbert S. Zuckerman, Structure theory for a class of convolution algebras .................................. 913

Amnon Jakimovski, Some Tauberian theorems . . . . . . . . . . . . . . . . . 943

C. T. Rajagopal, Simplified proofs of "Some Tauberian theorems" of Jakimovski................................

Paul Joseph Kelly, A congruence theorem for trees ................. 961

Robert Forbes McNaughton, Jr., On the measure of normal formulas...... 969

Richard Scott Pierce, Distributivity in Boolean algebras .............. 983

Calvin R. Putnam, Continuous spectra and unitary equivalence ......... 993

Marvin Rosenblum, Perturbation of the continuous spectrum and unitary

equivalence................................... 997

V. N. Singh, Certain generalized hypergeometric identities of the

Rogers-Ramanujan type.......................

Peter Swerling, Families of transformations in the function spaces $H^{p} \ldots \ldots 1015$ 